\title{
Thermistor Temperature Measurement Network with a Small Number of Wires
}

\author{
Vladimir V. Shaydurov ${ }^{\mathrm{a}, \mathrm{b}}$ and Anna A. Korneeva*a \\ ${ }^{a}$ Siberian Federal University \\ 79 Svobodny, Krasnoyarsk, 660041, Russia \\ ${ }^{b}$ Institute of Computational Modeling SB RAS \\ 50/44 Akademgorodok, Krasnoyarsk, 660036, Russia
}

Received 15.11.2018, received in revised form 15.01.2019, accepted 20.02.2019

The article proposes a new measuring circuit for temperature control at multiple points of the product using a sequence of thermistors and diodes with three connecting wires and two voltage sources. A mathematical model and a computational algorithm for calculating the resistance of thermistors are presented which consist in the formation and sequential solution of systems of linear algebraic equations for different ratios of voltages of two power sources. A model problem and the results of a computational experiment are considered.

Keywords: temperature measurement, thermistor network, mathematical model, numerical algorithm.

\section{Терморезисторная сеть измерения температуры с небольшим количеством проводов}

\author{
В.В. Шайдуров ${ }^{\mathbf{a}, \mathbf{0}}$, А.А. Корнеева ${ }^{\mathrm{a}}$ \\ ${ }^{a}$ Сибирский федеральный университет \\ Россия, 660041, Красноярск, пр. Свободньій, 79
} ${ }^{6}$ Институт вычислительного моделирования СО РАН Россия, 660036, Красноярск, Академгородок, 50/44

В статье предложена новая измерительная схема для контроля температуры на множестве точек изделия с помощью последовательности терморезисторов и диодов с

(C) Siberian Federal University. All rights reserved

This work is licensed under a Creative Commons Attribution-NonCommercial 4.0 International License (CC BY-NC 4.0).

* Corresponding author E-mail address: shaidurov04@mail.ru 
тремя соединительными проводами и двумя источниками напряжения. Представлень математическая модель и расчетный алгоритм вычисления сопротивления терморезисторов, состоящие в формировании и последовательном решении систем линейных алгебраических уравнений при разных соотношениях напряжений двух источников питания. Рассмотрена модельная задача и результаты вычислительного эксперимента.

Ключевые слова: измерение температуры, сеть терморезисторов, математическая модель, численный алгоритм.

\section{Введение}

Наличие большого количества соединительных проводов усложняет реализацию измерительной системы на контролируемом изделии, особенно на борту космического аппарата. Поэтому образцы измерительных сетей с небольшим количеством проводов активно используются для температурного анализа исследуемых объектов в труднодоступных, экстремальных или агрессивных средах [1-7]. В таких сетях поступление всего одного измерения для анализа большого количества данных может компенсироваться динамической вариацией источника питания и последующей компьютерной обработкой данных [7].

В статье представлена измерительная сеть с тремя соединительными проводами и двумя источниками напряжения: постоянного тока и с пилообразным сигналом. В сети измеряется только ток в одном из проводов, а требуемые температуры (зависящие от текущих сопротивлений термисторов [8-10]) рассчитываются специальным компьютерным алгоритмом обработки имеющейся информации. Источник напряжения пилообразного сигнала создает различные режимы питания, которые приводят к переключению состояния сети за счет перехода части диодов из закрытого состояния в открытое. Грубо говоря, закрытые диоды трансформируют исходную сеть в более простую [11], так что, по существу, одна и та же величина напряжения последовательно снимается с нескольких разных сетей. Полученные с их помощью искомые температурные данные можно считать вычисленными с определенной точностью либо использовать их как начальное приближение для последующего уточнения при требованиях повышенной точности.

\section{Принципиальная схема и расчетная модель измерительной сети}

Рассмотрим принципиальную схему на рис. 1. «Опорное» постоянное напряжение $E_{1}$ подается на последовательную цепь «делителя напряжения» из резисторов $R_{0}, R_{1}, \ldots, R_{n}$. Пилообразное напряжение $E_{2}$ подается на общий контакт $n$ пар диод-терморезисторов $D_{1}+T_{1}, \ldots, D_{n}+T_{n}$, которые подключены между соседними резисторами «делителя напряжения».

Значения сопротивлений терморезисторов являются искомыми величинами. Для их определения используются два заданных значения $E_{1}, E_{2}$ и измеряемый ток $I$ по показаниям амперметра $A$. Затем сопротивление каждого терморезистора пересчитывается в его температуру с учетом графика строгой монотонной зависимости сопротивления от температуры. При наличии вольтметра измерение тока можно заменить измерением падения напряжения на резисторе $R_{0}$ с исключением из цепи амперметра $A$.

Геометрически реализация схемы осуществляется путем компактного последовательного расположения троек $D_{k}+T_{k}+R_{k}$, соединяемых с соседями двумя проводниками по всей длине 


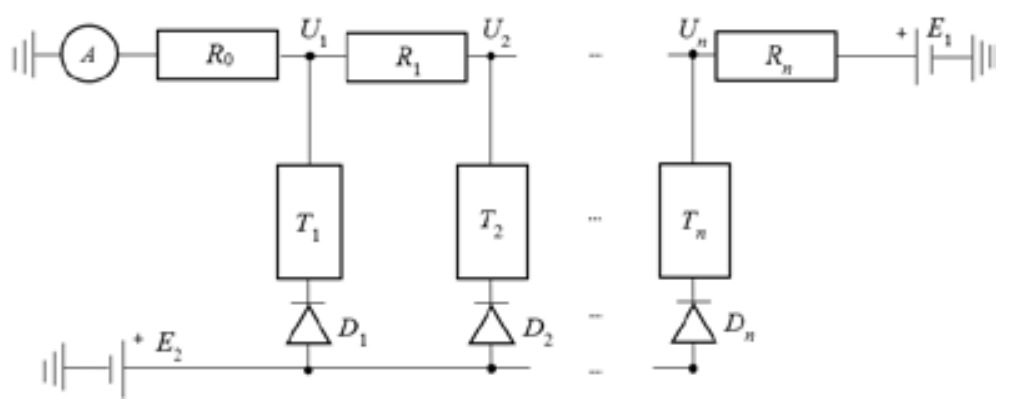

Рис. 1. Измерительная схема

Fig. 1. Measuring circuit

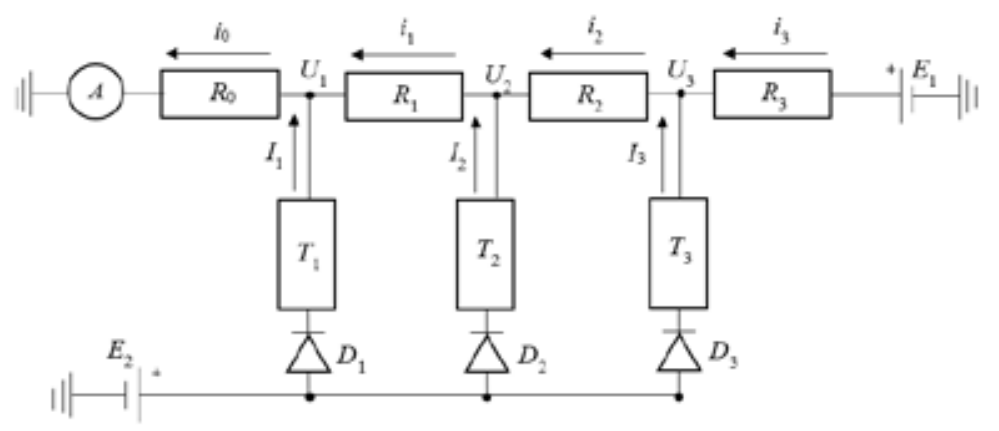

Рис. 2. Направления токов в схеме с тремя терморезисторами

Fig. 2. Directions of currents in the circuit with three thermistors

цепи, располагаемой по площади или объему контролируемого изделия. Третий провод используется только в конце измерительной сети для подачи опорного напряжения. Использование общего заземления (например, на металлоконструкциях) может вообще устранить его протяженную реализацию.

Для упрощения обозначим численные значения сопротивлений резисторов $R_{k}$, диодов $D_{k}$ и терморезисторов $T_{k}$ одноименными символами с этими элементами схемы. Для формирования математической модели зафиксируем эти сопротивления и напряжения $E_{1}, E_{2}$. Ток, входящий в $k$-тый узел со стороны $k+1$-го узла, обозначим через $i_{k}$, а ток со стороны $k$-той измерительной пары $D_{k}+T_{k}$ - через $I_{k}$. Величина $i_{0}$ соответствует току, выходящему из первого узла в сторону заземления. Выбор знаков поясняется на рис. 2 для трех измерительных пар. Выпишем первый закон Кирхгофа для токов в узлах с номерами $k=1, \ldots, n[11]$ :

$$
\begin{gathered}
-i_{0}+i_{1}+I_{1}=0, \\
-i_{1}+i_{2}+I_{2}=0, \\
\ldots \\
-i_{n-1}+i_{n}+I_{n}=0 .
\end{gathered}
$$

Заменим токи напряжениями по закону Ома и приведем подобные члены: 


$$
\left\{\begin{array}{c}
U_{1}\left(\frac{1}{R_{0}}+\frac{1}{T_{1}+D_{1}}+\frac{1}{R_{1}}\right)-U_{2} \frac{1}{R_{1}}=E_{2} \frac{1}{T_{1}+D_{1}}, \\
U_{2}\left(\frac{1}{R_{1}}+\frac{1}{T_{2}+D_{2}}+\frac{1}{R_{2}}\right)-U_{1} \frac{1}{R_{1}}-U_{3} \frac{1}{R_{2}}=E_{2} \frac{1}{T_{2}+D_{2}}, \\
\cdots \\
U_{n}\left(\frac{1}{R_{n-1}}+\frac{1}{T_{n}+D_{n}}+\frac{1}{R_{n}}\right)-U_{n-1} \frac{1}{R_{n-1}}=E_{2} \frac{1}{T_{n}+D_{n}}+E_{1} \frac{1}{R_{n}} .
\end{array}\right.
$$

В результате получилась система линейных алгебраических уравнений метода узловых потенциалов с трехдиагональной матрицей для определения неизвестных $U_{1}, U_{2}, \ldots, U_{n}$. Матрица неразложима, имеет диагональное преобладание, причем строгое в первой и последней строках [12]. Поэтому она невырождена и при положительных $E_{1}, E_{2}$ все потенциалы $U_{1}, U_{2}, \ldots, U_{n}$ тоже положительны [12]. Вообще говоря, все уравнения системы (2) можно записать в однородной форме

$$
-U_{k-1} \frac{1}{R_{k-1}}+U_{k}\left(\frac{1}{R_{k-1}}+\frac{1}{T_{k}+D_{k}}+\frac{1}{R_{k}}\right)-U_{k+1} \frac{1}{R_{k}}=E_{2} \frac{1}{T_{k}+D_{k}} \quad \text { для } \quad k=1, \ldots, n
$$

с учетом «краевых значений» $U_{0}=0$ и $U_{n+1}=E_{1}$.

Проиллюстрируем основную идею в частном случае с тремя терморезисторами, представленном на рис. 2. В этом случае система (2) записывается в виде

$$
\left\{\begin{array}{l}
U_{1}\left(\frac{1}{R_{0}}+\frac{1}{T_{1}+D_{1}}+\frac{1}{R_{1}}\right)-E_{2} \frac{1}{T_{1}}-U_{2} \frac{1}{R_{1}}=0 \\
U_{2}\left(\frac{1}{R_{1}}+\frac{1}{T_{2}+D_{2}}+\frac{1}{R_{2}}\right)-U_{1} \frac{1}{R_{1}}-E_{2} \frac{1}{T_{2}}-U_{3} \frac{1}{R_{2}}=0, \\
U_{3}\left(\frac{1}{R_{2}}+\frac{1}{T_{3}+D_{3}}+\frac{1}{R_{3}}\right)-U_{2} \frac{1}{R_{2}}-E_{2} \frac{1}{T_{3}}-E_{1} \frac{1}{R_{3}}=0 .
\end{array}\right.
$$

Процесс нахождения неизвестных сопротивлений терморезисторов $T_{1}, T_{2}$ и $T_{3}$ состоит из последовательного рассмотрения трех состояний измерительной схемы: $U_{1}<E_{2}<U_{2}, U_{2}<E_{2}<U_{3}$ и $E_{2}>U_{3}$.

При $U_{1}<E_{2}<U_{2}$ диод $D_{1}$ открыт, а диоды $D_{2}, D_{3}$ находятся в закрытом состоянии. Рассмотрим «идеальные» диоды, у которых сопротивление открытого состояния близко к нулю: $D_{1}=0$, а у закрытого состояния проводимость близка к нулю: $D_{2}=+\infty$ и $D_{3}=+\infty$. Тогда система (4) принимает вид

$$
\left\{\begin{array}{l}
U_{1}\left(\frac{1}{R_{0}}+\frac{1}{T_{1}}+\frac{1}{R_{1}}\right)-E_{2} \frac{1}{T_{1}}-U_{2} \frac{1}{R_{1}}=0 \\
U_{2}\left(\frac{1}{R_{1}}+\frac{1}{R_{2}}\right)-U_{1} \frac{1}{R_{1}}-U_{3} \frac{1}{R_{2}}=0 \\
U_{3}\left(\frac{1}{R_{2}}+\frac{1}{R_{3}}\right)-U_{2} \frac{1}{R_{2}}-E_{1} \frac{1}{R_{3}}=0
\end{array}\right.
$$

Из первого уравнения следует, что 


$$
T_{1}=\left(U_{1}-E_{2}\right) /\left(\frac{U_{2}}{R_{1}}-U_{1}\left(\frac{1}{R_{0}}+\frac{1}{R_{1}}\right)\right) .
$$

$U_{1}$ известно, поскольку является (измеренным) падением напряжения на фиксированном резисторе $R_{0}$. $U_{2}$ при «разомкнутых» ветвях $D_{2}+T_{2}$ и $D_{3}+T_{3}$ определяется делителем напряжения между потенциалами $E_{1}$ и $U_{1}$ :

$$
U_{2}=\left(E_{1}-U_{1}\right) R_{1} /\left(R_{1}+R_{2}+R_{3}\right) .
$$

В итоге, все величины в (6) известны и однозначно определяют $T_{1}$.

При открытых диодах $D_{1}$ и $D_{2}\left(U_{2}<E_{2}<U_{3}\right)$ система (4) принимает вид

$$
\left\{\begin{array}{l}
U_{1}\left(\frac{1}{R_{0}}+\frac{1}{T_{1}}+\frac{1}{R_{1}}\right)-E_{2} \frac{1}{T_{1}}-U_{2} \frac{1}{R_{1}}=0 \\
U_{2}\left(\frac{1}{R_{1}}+\frac{1}{T_{2}}+\frac{1}{R_{2}}\right)-U_{1} \frac{1}{R_{1}}-E_{2} \frac{1}{T_{2}}-U_{3} \frac{1}{R_{2}}=0 \\
U_{3}\left(\frac{1}{R_{2}}+\frac{1}{R_{3}}\right)-U_{2} \frac{1}{R_{2}}-E_{1} \frac{1}{R_{3}}=0 .
\end{array}\right.
$$

Из второго уравнения следует, что

$$
T_{2}=\left(U_{2}-E_{2}\right) /\left(\frac{U_{1}}{R_{1}}+\frac{U_{3}}{R_{2}}-U_{2}\left(\frac{1}{R_{1}}+\frac{1}{R_{2}}\right)\right) .
$$

В правой части нам известно (новое измеренное) $U_{1}$. Поскольку $T_{1}$ ранее определено, из первого уравнения определяем $U_{2}$

$$
U_{2}=R_{1} U_{1}\left(\frac{1}{R_{0}}+\frac{1}{T_{1}}+\frac{1}{R_{1}}\right)-E_{2} \frac{R_{1}}{T_{1}}
$$

$U_{3}$ при «разомкнутой» ветви $D_{3}+T_{3}$ определяется делителем напряжения между потенциалами $E_{1}$ и $U_{2}$ :

$$
U_{3}=\left(E_{1}-U_{2}\right) R_{2} /\left(R_{2}+R_{3}\right)
$$

В итоге, все величины в (8) известны и однозначно определяют $T_{2}$.

При $E_{2}>U_{3}$ все диоды открыты. Тогда из третьего уравнения системы (4) вытекает, что

$$
T_{3}=\left(U_{3}-E_{2}\right) /\left(\frac{U_{2}}{R_{2}}+\frac{E_{1}}{R_{3}}-U_{3}\left(\frac{1}{R_{2}}+\frac{1}{R_{3}}\right)\right) .
$$

Поскольку $T_{1}$ и $T_{2}$ уже определены, из первого уравнения определяем $U_{2}$ по формуле (9), а из второго уравнения вычисляем

$$
U_{3}=R_{2} U_{2}\left(\frac{1}{R_{1}}+\frac{1}{T_{2}}+\frac{1}{R_{2}}\right)-U_{1} \frac{R_{2}}{R_{1}}-E_{2} \frac{R_{2}}{T_{2}} .
$$

Подставляя их в (10), однозначно определяем величину $T_{3}$. 
В случае большего количества троек $D_{k}+T_{k}+R_{k}$ вычисление $T_{k}$ осуществляется аналогично по нарастанию индекса $k$ от 1 до $n$ в момент подачи напряжения $E_{2} \in\left(U_{k}, U_{k-1}\right)$ для открывания диодов $D_{1}, \ldots, D_{k}=0$ при закрытых диодах $D_{k+1}, \ldots, D_{n}=+\infty$. К этому моменту считаются вычисленными величины $T_{1}, \ldots, T_{k-1}$. Тогда из уравнения (3) получаем

$$
T_{k}=\left(U_{k}-E_{2}\right) /\left(\frac{U_{k-1}}{R_{k-1}}+\frac{U_{k+1}}{R_{k}}-U_{k}\left(\frac{1}{R_{k-1}}+\frac{1}{R_{k}}\right)\right) .
$$

Участвующие в этой формуле $U_{k-1}$ и $U_{k}$ вычисляются из уравнений (3) с нулевыми $D_{i}$ :

$$
U_{i}=R_{i-1}\left(-\frac{U_{i-2}}{R_{i-2}}+U_{i-1}\left(\frac{1}{R_{i-2}}+\frac{1}{T_{i-1}}+\frac{1}{R_{i-1}}\right)-\frac{E_{2}}{T_{i-1}}\right) \quad \text { для } i=2, \ldots, k .
$$

А $U_{k-1}$ вычисляется из других уравнений (3) с бесконечными $D_{i}$, что численно реализуется в виде делителя напряжения между $E_{1}$ и $U_{k}$ :

$$
U_{k+1}=\left(E_{1}-U_{k}\right) R_{k} /\left(R_{k}+\ldots+R_{n+1}\right) .
$$

\section{Вычислительный эксперимент}

Рассмотрим модельную задачу с «идеальными»диодами. Пусть $E_{1}=5 \mathrm{~B}, R_{0}=R_{1}=R_{2}=R_{3}=900$ Ом, $T_{1}=2000$ Ом, $T_{2}=3000$ Ом, $T_{3}=4000$ Ом. Для расчета параметров электрической цепи применяли описанный метод узловых потенциалов. В тестовых расчетах использовались «идеальные» диоды в следующем смысле: у открытого диода сопротивление равно нулю, а у закрытого проводимость равна нулю. Отметим, что эти условия плохо выполняются при незначительном падении напряжения на реальном диоде. Поэтому при измерениях следует избегать ситуаций, близких к точкам переключения диодов.

Сначала проведем вычисление узловых потенциалов при изменении постоянного напряжения $E_{2}$ в интервале $[0.2,5.0]$ с шагом 0.2. Полученные значения представлены в табл. 1-4.

В качестве исходных данных для работы алгоритма были выбраны три ситуации с разными состояниями диодов:

1) состояние 8: $E_{1}=5.0 \mathrm{~B}, E_{2}=1.8 \mathrm{~B}, U_{1}=1.39 \mathrm{~B}$;

Таблица 1. Все диоды находятся в закрытом состоянии

Table 1. All diodes are in the closed state

\begin{tabular}{|c|c|c|c|c|}
\hline Такт & $E_{2}, \mathrm{~B}$ & $U_{1}, \mathrm{~B}$ & $U_{2}, \mathrm{~B}$ & $U_{3}, \mathrm{~B}$ \\
\hline 0 & 0.20 & 1.25 & 2.50 & 3.75 \\
\hline 1 & 0.40 & 1.25 & 2.50 & 3.75 \\
\hline 2 & 0.60 & 1.25 & 2.50 & 3.75 \\
\hline 3 & 0.80 & 1.25 & 2.50 & 3.75 \\
\hline 4 & 1.00 & 1.25 & 2.50 & 3.75 \\
\hline 5 & 1.20 & 1.25 & 2.50 & 3.75 \\
\hline
\end{tabular}

Таблица 2. Диод $D_{1}$ открыт, $D_{2}$ и $D_{3}$ закрыты

Table 2. Diode $D_{1}$ is open, $D_{2}$ и $D_{3}$ are closed

\begin{tabular}{|c|c|c|c|c|}
\hline Такт & $E_{2}, \mathrm{~B}$ & $U_{1}, \mathrm{~B}$ & $U_{2}, \mathrm{~B}$ & $U_{3}, \mathrm{~B}$ \\
\hline 6 & 1.40 & 1.29 & 2.53 & 3.76 \\
\hline 7 & 1.60 & 1.34 & 2.56 & 3.78 \\
\hline 8 & 1.80 & 1.39 & 2.59 & 3.80 \\
\hline 9 & 2.00 & 1.44 & 2.63 & 3.81 \\
\hline 10 & 2.20 & 1.49 & 2.66 & 3.83 \\
\hline 11 & 2.40 & 1.54 & 2.69 & 3.85 \\
\hline 12 & 2.60 & 1.59 & 2.73 & 3.86 \\
\hline
\end{tabular}


Таблица 3. Диоды $D_{1}$ и $D_{2}$ открыты, $D_{3}$ закрыт

Table 3. Diodes $D_{1}$ and $D_{2}$ are open, $D_{3}$ is closed

\begin{tabular}{|c|c|c|c|c|}
\hline Такт & $E_{2}, \mathrm{~B}$ & $U_{1}, \mathrm{~B}$ & $U_{2}, \mathrm{~B}$ & $U_{3}, \mathrm{~B}$ \\
\hline 13 & 2.80 & 1.65 & 2.77 & 3.88 \\
\hline 14 & 3.00 & 1.71 & 2.83 & 3.92 \\
\hline 15 & 3.20 & 1.77 & 2.91 & 3.95 \\
\hline 16 & 3.40 & 1.84 & 2.98 & 3.99 \\
\hline 17 & 3.60 & 1.90 & 3.05 & 4.02 \\
\hline 18 & 3.80 & 1.97 & 3.12 & 4.06 \\
\hline 19 & 4.00 & 2.04 & 3.19 & 4.09 \\
\hline
\end{tabular}

Таблица 5. Результаты вычислительного эксперимента

Table 5. Results of the computational experiment

\begin{tabular}{|l|c|c|c|}
\hline \multicolumn{1}{|c|}{ Открытые диоды } & $U_{2}, \mathrm{~B}$ & $U_{3}, \mathrm{~B}$ & $T_{i}$, Ом \\
\hline$D_{1}$ & 2.59 & 3.79 & $T_{1}=2000.20$ \\
\hline$D_{1}, D_{2}$ & 2.91 & 3.95 & $T_{2}=3000.29$ \\
\hline$D_{1}, D_{2}, D_{3}$ & 3.42 & 4.25 & $T_{3}=4000.55$ \\
\hline
\end{tabular}

2) состояние 15: $E_{1}=5.0 \mathrm{~B}, E_{2}=3.2 \mathrm{~B}, U_{1}=1.77 \mathrm{~B}$;

3) состояние 22: $E_{1}=5.0 \mathrm{~B}, E_{2}=4.6 \mathrm{~B}, U_{1}=2.24 \mathrm{~B}$.

Полученные результаты работы алгоритма представлены в табл. 5.

Ошибка восстановления сопротивлений $T_{1}, T_{2}, T_{3}$ составила менее $0.014 \%$.

\section{Заключение}

Ясно, что в реальных схемах вольтамперные характеристики диодов не столь идеальны. Поэтому ошибка восстановления сопротивления термисторов получается тем больше, чем больше отклонения характеристик диодов от идеального состояния, т.е. чем больше выражения

$$
D_{k}^{\mathrm{op}} / T_{k} \text { и } T_{k} / D_{k}^{\mathrm{cl}}
$$

для открытого $D_{k}^{\text {op }}$ и закрытого $D_{k}^{\text {cl }}$ состояний диодов соответственно. Точность будет тем выше, чем меньше величины (15), которые необходимо учитывать при подборе реальных элементов измерительной схемы.

Снижение точности может происходить при большом числе троек $D_{k}+T_{k}+R_{k}$, когда ненулевые обратные токи суммарно могут дать существенный вклад в погрешность изложенного алгоритма. Тогда изложенный алгоритм применяется для получения начального приближения для решения полной системы нелинейных алгебраических уравнений (3), в которых сопротивления $D_{k}$, в принципе, не только зависят от направления тока, но и могут нелинейно зависеть от амплитуды напряжения и даже от температуры. В таком случае для решения нелинейных 
систем уравнений, получающихся при каждом конкретном режиме включения диодов, должна быть известна (нелинейная) температурная вольтамперная характеристика используемых диодов.

Исследование выполнено при финансовой поддержке Российского фонда фундаментальных исследований, Правительства Красноярского края, Красноярского краевого фонда науки в рамках научного проекта: № 18-47-242005 «Создание эффективных распределенных сетей температурных датчиков для бортовой аппаратуры спутников».

\section{Список литературы}

[1] Линевег Ф. Измерение температур в технике. М.: Металлургия, 1980. 544 с. [Lineveg F. Temperature measurement in the technique. M.: Metallurgiya, 1980. 544 p. (in Russian)].

[2] Васильев Е.Н., Деревянко В.А. Анализ эффективности применения термоэлектрических модулей в системах охлаждения радиоэлементов. Вестник СибГАУ, 2013, 4 (50), 9-13 [Vasil'ev E.N., Derevyanko V.A. Analysis of the effectiveness of the use of thermoelectric modules in cooling systems of radioelements. Vestnik SibSAU, 2013, 4 (50), 9-13 (in Russian)].

[3] Деревянко В.А., Латыпов А.Ф. Восстановление распределения температуры среды в двумерной области томографическим методом по результатам измерений электрического сопротивления ориентированных проводников. Вычислительные технологии, 2018, 23 (3), 31-38 [Derevyanko V.A., Latypov A.F. Recovery of the temperature distribution of the medium in the twodimensional domain by the tomographic method from the results of measurements of the electrical resistance of oriented conductors. Computational Technologies, 2018, 23 (3), 31-38 (in Russian)]

[4] Корнеева А.А., Шайдуров В.В. Численный анализ температурных данных с пленочных резисторов электронных плат. Вычислительные технологии, 2017, 22 (3), 32-44. [Korneeva A.А., Shaydurov V.V. Numerical analysis of temperature data from film resistors of electronic boards. Computational Technologies, 2017, 22 (3), 32-44 (in Russian)]

[5] Шайдуров В.В., Корнеева А.А. Численный метод анализа нелокальных данных с пленочных терморезисторов электронных плат. Сибирские электронные математические известия, 2017, 14, 914-926. [Shaydurov V.V., Korneeva A.A. Numerical method for the analysis of nonlocal data from film thermistors of electronic boards. Siberian Electronic Mathematical Reports, 2017, 14, 914-926 (in Russian)].

[6] Korneeva A., Shaydurov V. Computational algorithms for analysis of data from thin-film thermoresistors on a radio-electronic printed circuit board. Conference Proceedings of American Institute of Physics, 2016, 1759, Art. 020048.

[7] Caltagirone J.P. Reseau maille de conducteurs electriques, notamment pour mesure de temperatures. France: Demande de brevet d'invention № 2315689, Intern. Classification G01K7/04. 1977 [Caltagirone J.P. Mesh network of electrical conductors, especially for measuring temperatures. France: Patent application No. 2315689, Intern. Classification G01K7/04. 1977 (in French)].

[8] Wood S.D., Mangum B.W., Filliben J.J., Tillett S.B. An investigation of the stability of thermistors. J. Research of the National Bureau of Standards, 1978, 83 (3), 247-263.

[9] Precise and reliable measurement, optimisation and management. Germany: Heraeus Sensor Technology, 2017. 38 p. 
[10] Ядевич А.И. Тонкопленочные платиновые чувствительные элементы для термометров сопротивления. Мир измерений, 2010, 3, $52-55$ [Yadevich A.I. Platinum thin film sensors for resistance thermometers, Measurement World, 2010, 3, 52-55 (in Russian)].

[11] Нейман Л.Р., Демирчян К.С. Теоретические основы электротехники. Том І. Л.: Энергоиздат, 1981. 536 c. [Neiman L.R., Demirchyan K.S. Theoretical foundations of electrical engineering. Vol. 1. L: Energoizdat, 1981, 536 p. (in Russian)].

[12] Воеводин В.В., Кузнецов Ю.А. Матрицы и вычисления. М.: Наука, 1984. 320 с. [Voevodin V.V., Kuzetsov Yu.A. Matrices and computations. M.: Nauka, 1984. 320 p. (in Russian)]. 